\title{
Equivalence between a Gravity Field and an Unruh Acceleration Temperature Field as a Possible Clue to "Dark Matter"
}

\author{
Eugene Terry Tatum ${ }^{1}$, U. V. S. Seshavatharam ${ }^{2}$ \\ ${ }^{1} 760$ Campbell Ln. Ste. 106 \#161, Bowling Green, KY 42104, USA \\ ${ }^{2}$ Honorary Faculty, I-SERVE, Hyderabad, India \\ Email: ett@twc.com, seshavatharam.uvs@gmail.com
}

How to cite this paper: Tatum, E.T. and Seshavatharam, U.V.S. (2018) Equivalence between a Gravity Field and an Unruh Acceleration Temperature Field as a Possible Clue to "Dark Matter". Journal of Modern Physics, 9, 1568-1572.

https://doi.org/10.4236/jmp.2018.98098

Received: June 28, 2018

Accepted: July 15, 2018

Published: July 18, 2018

Copyright $\odot 2018$ by authors and Scientific Research Publishing Inc. This work is licensed under the Creative Commons Attribution International License (CC BY 4.0).

http://creativecommons.org/licenses/by/4.0/

\begin{abstract}
Einstein's equivalence principle allows one to compare the magnitudes of a gravitational acceleration field with the magnitudes of a field of Unruh acceleration temperatures. The validity of such a comparison is demonstrated by using it to derive the effective Hawking black body radiation at a Schwarzschild black hole horizon. One can then extend the black hole thought experiment to a Hawking-Unruh temperature equation expressed in terms of the Schwarzschild radius. This follows an inverse radius law rather than an inverse radius-squared law. Following a brief discussion of current theoretical failures to explain galactic rotation curves, the Unruh acceleration temperature equations are brought together to show how a rotating supermassive black hole galactic system should follow an inverse radius rule of centripetal gravitational force and centripetal acceleration. This result appears to indicate that galactic observations currently attributed to dark matter may in part be attributed to classical Newtonian dynamics superimposed on a relativistic rotating system powered by a supermassive black hole.
\end{abstract}

\section{Keywords}

Dark Matter, Unruh Effect, Unruh Temperature, Supermassive Black Holes, Galactic Rotation Curves, Equivalence Principle, Hawking Radiation

\section{Introduction and Background}

It is well-known that an inertial reference frame within a gravity field can be treated as equivalent to an accelerating reference frame (Einstein's equivalence principle). This is why a gravity field can be represented entirely by gamma acceleration vectors. It is also well-known that an accelerating observer or detector 
in a vacuum field will observe a black body radiation spectrum appearing to originate in-line with the direction of acceleration. This is most commonly known as the Unruh effect, and the black body temperature is known as the Unruh acceleration temperature. The Unruh acceleration temperature is given by the following formula [1]:

$$
T \cong\left(\frac{\hbar}{2 \pi c k_{B}}\right) a
$$

wherein symbol "a" represents the acceleration, and all symbols in the brackets are known constants.

Of particular interest for this discussion is that one could imagine a field of Unruh acceleration temperatures around an isolated gravitating body, with its relative magnitudes corresponding in direct proportion to the gamma field magnitudes. For instance, if one were to place two identical Schwarzschild black holes of mass $M$ at a separation distance of their Schwarzschild radii, they would experience a net attractive force per unit mass (i.e., a forward acceleration " $a$ ") of $\left(\frac{c^{4}}{4 G M}\right)$.Thus, they would each experience an Unruh acceleration temperature of $\left(\frac{\hbar c^{3}}{8 \pi k_{B} G M}\right)$ at the horizon of the opposing black hole. This particular result represented by

$$
T \cong \frac{\hbar c^{3}}{8 \pi k_{B} G M}
$$

is identical to the Hawking temperature derivation now known as Hawking radiation [2]. Hawking radiation can be considered the black hole black body radiation taking place at or very near every black hole horizon. Thus, temperature $T$ in Equation (2) is also sometimes referred to as the Hawking-Unruh temperature [3].

It is also valid to use the Schwarzschild formula and substitute $\left(\frac{R c^{2}}{2 G}\right)$ for $M$, giving the equivalent Hawking-Unruh temperature in the form of

$$
T \cong \frac{\hbar c}{4 \pi k_{B} R}
$$

wherein $R$ represents the Schwarzschild radius and the other symbols in the right-hand term are known constants.

By Equation (1) we can imagine a proportional equivalency between the magnitudes of an Unruh acceleration temperature field and the gamma magnitudes of a gravitational field. In Equation (3) we see indirect proportionality between the magnitude of the Unruh acceleration temperature field at a black hole's horizon and the magnitude of its Schwarzschild radius $R$. Of particular note is that these relationships can only be true if the gravitational field around a black hole of any size does not follow an inverse $R$-squared law but rather an inverse $R$ law! 


\section{Discussion}

It has only recently become evident that all, or nearly all, galaxies have a central supermassive black hole. However, for a number of decades, the rotation curves of galaxies have been quite puzzling [4] [5] [6]. Their plate-like stellar rotation was a clear indication that the classical inverse $R$-squared gravitational law of our solar system could not easily be extended to galaxies. These aberrant galactic rotations have long been known to act more as if stellar centripetal forces (and their associated accelerations) vary inversely with radius distance from the galactic center, rather than the expected radius distance squared.

Over the last few decades, two basic theoretical approaches have been attempted to address this paradox [7] [8]. By far the most popular approach has been to suggest a halo-like distribution of non-visible (i.e., "dark"), non-baryonic, cold matter which only interacts with ordinary visible matter by gravitational attraction. However, numerous varied and highly creative observational studies to detect and determine the exact nature of dark matter, including how it revises the Standard Particle Model, have failed in this regard. The second theoretical approach has been to find a reasonable modification of Newton's law of gravity in order to explain the aberrant galactic rotation. This search for a theory of Modified Newtonian Dynamics (MOND) has met with limited success [9], mostly by an ad hoc search for new gravitational equations, effects of which are designed to become noticeable only at the extremely small stellar centripetal accelerations within the outer two-thirds of the galactic disc.

Now recent theoretical approaches [10] [11] [12], as well as computer-generated Gaia star map analysis [13], strongly suggest that a new type of matter outside of the Standard Particle Model, or an ad hoc modification of Newtonian dynamics, may be completely unnecessary. The report on the Gaia billion-star map study by Enbang Li concluded that the flat galactic rotation curve of the Milky Way galaxy can be entirely explained without the need for a dark matter halo! Erik Verlinde's "emergent gravity" theory and Flat Space Cosmology [Tatum, et al. (2018)] provide support for inertial effects of galactic visible matter entropy as being largely responsible for "dark matter" observations. The July 2018 Journal of Modern Physics paper entitled "A Potentially Useful Dark Matter Index" [14] references four very recent observational studies which appear to support this concept of a possible link between "dark matter" and inertial effects of galactic gravitational entropy.

Furthermore, it occurs to this author that we have been thinking about a rotating galaxy in the wrong way. Rather than trying to impose classical Keplerian and Newtonian solar system model kinematics and dynamics, we should think of galaxies as a rotating supermassive black hole ( $S M B H)$ system. If we think of galactic evolution as beginning with fast-spinning quasars and blazars, we are probably better-equipped to think of modern co-moving galaxies as being relatively quiescent former dynamos which began with extremely powerful organizing magnetic fields. For this reason, perhaps, modern galaxies appear to behave 
more like a spinning flywheel than a solar system.

Furthermore, and perhaps most importantly, it should not surprise us if dynamic SMBH systems have long-since locked in their plate-like stellar rotations with an inverse radius rule along the lines of Equation (3). By combining Equations (1) and (3), which can both be applied, as shown, to black holes (specifically SMBHs in this case), we get

$$
\left(\frac{\hbar}{2 \pi c k_{B}}\right) a \cong \frac{\hbar c}{4 \pi k_{B} R}
$$

which simplifies to

$$
a \cong \frac{c^{2}}{2 R}
$$

One can see that this is a centripetal acceleration formula of the type $a_{c} \cong \frac{v^{2}}{R}$, wherein rotational velocity $v \cong \frac{c}{\sqrt{2}}$ is a constant at horizon radius $R$.

Thus, we have what appears to be classical Newtonian dynamics superimposed on a relativistic rotating galactic system powered by a SMBH.

\section{Summary and Conclusions}

Einstein's equivalence principle allows one to compare the magnitudes of a gravitational acceleration field with the magnitudes of a field of Unruh acceleration temperatures. The validity of such a comparison is demonstrated by using it to derive the effective Hawking black body radiation at a Schwarzschild black hole horizon. One can then extend the black hole thought experiment to a Hawking-Unruh temperature equation expressed in terms of the Schwarzschild radius. This follows an inverse radius law rather than an inverse radius-squared law. Following a brief discussion of current theoretical failures to explain galactic rotation curves, the Unruh acceleration temperature equations are brought together to show how a rotating supermassive black hole galactic system should follow an inverse radius rule of centripetal gravitational force and centripetal acceleration. This result appears to indicate that galactic observations currently attributed to dark matter may in part be attributed to classical Newtonian dynamics superimposed on a relativistic rotating system powered by a supermassive black hole.

\section{Dedications and Acknowledgements}

This paper is dedicated to Dr. Stephen Hawking and Dr. Roger Penrose for their groundbreaking work on black holes and their possible application to cosmology. Dr. Tatum also thanks Dr. Rudolph Schild of the Harvard Center for Astrophysics for his past support and encouragement.

\section{References}

[1] Unruh, W.G. (2001) Black Holes, Dumb Holes, and Entropy. In: Callender, C., Ed., 
Physics Meets Philosophy at the Planck Scale, Cambridge University Press, 152-173, Eq. 7.6. https://doi.org/10.1017/CBO9780511612909.008

[2] Hawking, S. (1976) Physical Review D, 13, 191-197. https://doi.org/10.1103/PhysRevD.13.191

[3] Alsing, P.M. and Milonni, P.W. (2004) American Journal of Physics, 72, 1524. arXiv:quant-ph/0401170.

[4] Rubin, V.C., Thonnard, N. and Ford, W.K. (1978) The Astrophysical Journal Letters, 225, L107-L111.

[5] Salucci, P. and De Laurentis, M. (2012) Proceedings of Science (DSU 2012). arXiv:1302.2268.

[6] Salucci, P., et al. (2007) Monthly Notices of the Royal Astronomical Society, 378, 41-47. arXiv:astro-ph/0703115. https://doi.org/10.1111/j.1365-2966.2007.11696.x

[7] McGaugh, S. (2014) Canadian Journal of Physics, 93, 250-259. arXiv:1404.7525

[8] Milgrom, M. (1983) Astrophysical Journal, 270, 365-370.

[9] Kroupa, P., Pawlowski, M. and Milgrom, M. (2012) International Journal of Modern Physics D, 21, 1230003.arXiv:1301.3907

[10] Verlinde, E. (2011) Journal of High Energy Physics, 4, 29-55. arXiv:1001.0785v1 [hep-th] https://doi.org/10.1007/JHEP04(2011)029

[11] Verlinde, E. (2016) Emergent Gravity and the Dark Universe. arXiv:1611.02269v2 [hep-th]

[12] Tatum, E.T. and Seshavatharam, U.V.S. (2018) Journal of Modern Physics, 9, 1469-1483. https://doi.org/10.4236/jmp.2018.98091

[13] Li, E. (2018) Modelling Mass Distribution of the Milky Way Galaxy Using Gaia's Billion-Star Map. https://arxiv.org/ftp/arxiv/papers/1612/1612.07781.pdf

[14] Tatum, E.T. (2018) Journal of Modern Physics, 9, 1564-1567. 